\title{
Propriedades físicas de misturas para sherbet de mangaba
}

\author{
Alessandra Lopes de Oliveira( ${ }^{(1)}$, Milton Guilherme Flora da Silva ${ }^{(1)}$, Paulo José do Amaral Sobral(1), \\ Carlos Augusto Fernandes de Oliveira(1) e Ana Mônica Quinta Barbosa Habitante(1)
}

(1)Universidade de São Paulo, Fac. de Zootecnia e Engenharia de Alimentos, Dep. de Engenharia de Alimentos, Av. Duque de Caxias Norte, 225, Caixa Postal 23, CEP 13635-900 Pirassununga, SP. E-mail: alelopes@usp.br

\begin{abstract}
Resumo - O objetivo deste trabalho foi avaliar a viscosidade, a quantidade de água não congelada $\left(\mathrm{w}_{\mathrm{nc}}\right)$ e a temperatura de transição vítrea da fração maximamente crioconcentrada (Tg’) de misturas para sherbet, e a incorporação de ar (IAr) dos sherbets, com diferentes concentrações de goma guar (0,2\% a 0,5\%) e polpa de mangaba (21,1\%, 25,8\% e 28,6\%). A viscosidade aparente variou de 85,8 a 286,1 cP, aumentando com as concentrações de goma guar e mangaba. As análises térmicas com calorímetro diferencial de varredura mostraram que a $\mathrm{w}_{\mathrm{nc}}$ variou entre $12 \%$ e $20 \%$, diminuindo com o aumento da concentração de polpa. A Tg' variou de $-57,65^{\circ} \mathrm{C}$ a $-53,45^{\circ} \mathrm{C}$ e não apresentou diferença entre as amostras. A IAr foi de 20,01\% a 40,59\%. Sherbets com a mesma concentração de polpa apresentaram valores diferentes desta variável, mas uma relação entre o aumento da quantidade de goma guar e maior IAr foi observada somente para os sherbets com 25,8\% de polpa. O estabilizante interfere na viscosidade da mistura e IAr, e a concentração de polpa influencia as propriedades térmicas. O aumento de IAr proporciona melhor maciez ao produto. Maior quantidade de polpa diminui a $\mathbf{w}_{\mathrm{nc}}$, assim o produto terá menos água disponível para migrar e formar cristais de gelo maiores durante as oscilações na temperatura, diminuindo a qualidade do produto.
\end{abstract}

Termos para indexação: sorvete, viscosidade, incorporação de ar, água não congelada, calorimetria diferencial de varredura.

\section{Physical properties of mixes to mangaba sherbet}

\begin{abstract}
Viscosity, unfrozen water $\left(\mathrm{w}_{\mathrm{nc}}\right)$ and glass transition temperature of the maximally freeze-concentrated material (Tg') of sherbet mixtures and the overrum of the sherbets with different concentrations of guar gum (from $0.2 \%$ to $0.5 \%$ ) and mangaba pulp (21.1\%, $25.8 \%$ and $28.5 \%$ ) were evaluated. Apparent viscosity ranged from 85.8 to $286.1 \mathrm{cP}$, increasing with guar gum concentration and mangaba pulp. Thermal analysis using differential scanning calorimetry of sherbets mixture showed that $\mathrm{w}_{\mathrm{nc}}$ was from $12 \%$ to $20 \%$, decreasing with the increasing of mangaba pulp concentration. The Tg' ranged from $-57.65^{\circ} \mathrm{C}$ to $-53.45^{\circ} \mathrm{C}$ and was not different between the samples. The overrum was from $20.1 \%$ to $40.59 \%$. Sherbets containing the same pulp concentration presented different values of this variable, however a relationship between the increasing of guar gum concentrations and the air incorporation was observed only for sherbets with $25.8 \%$ of mangaba pulp. The stabilizer modified the viscosity of the mixture and overrum and the pulp concentration influenced thermal properties. Higher overrum is better for product smoothness. Higher pulp concentration decreases the $\mathrm{w}_{\mathrm{nc}}$ so, the product concentrates less water available to form great ices during the changes in the temperature which may cause losses in the product quality.
\end{abstract}

Index terms: ice cream, viscosity, overrum, unfrozen water, differential scanning calorimetry.

\section{Introdução}

Sherbet é um produto congelado à base de água, açúcares, frutas ou aromatizantes de frutas, sólidos do leite, estabilizante e corantes, quando necessários. Esse produto contém de $1 \%$ a $2 \%$ de gordura do leite, não sendo permitida outra gordura, e de $2 \%$ a $5 \%$ de sólidos totais de leite (Anvisa, 1999). Similarmente ao sorvete, o sherbet é processado com maior quantidade de polpa de fruta, apresentando, portanto, sabor mais azedo. Além disso, apresenta menor overrum, isto é, menor incorporação de ar (de $25 \%$ a 50\%), maior teor de açúcar, menor ponto de fusão, e textura mais grosseira do que o sorvete (Marshall \& Arbuckle,1996).

Assim como no sorvete, a qualidade final do sherbet depende das propriedades funcionais dos ingredientes, das propriedades físicas da calda e do processo de fabricação. Os estabilizantes e a polpa de fruta influenciam as propriedades da mistura, tais como a viscosidade e a temperatura de fusão da calda, além das carac- 
terísticas físicas do produto final, como, por exemplo, a incorporação de ar (IAr).

A viscosidade da mistura influencia o tratamento térmico durante o processamento, afetando as transições de fases, como a fusão dos lipídeos e cristalização da água durante o batimento, e a IAr está relacionada com a textura do produto (Reid, 1998). A relação direta entre estas duas propriedades e o tempo de batimento influi na suavidade e maciez e, assim, na qualidade do produto durante o congelamento (Chang \& Hartel, 2002).

Vários estudos têm sido realizados sobre a influência do tipo e da concentração de estabilizantes no comportamento da viscosidade de sorvete (Kaya \& Tekin, 2001; Innocente et al., 2002) e sobre o crescimento de cristais de gelo e textura do produto final (Hartel, 1996; Marshall \& Arbuckle, 1996). A influência da concentração de estabilizantes que interagem com diferentes constituintes de sobremesas lácticas congeladas também foi estudada (Wittinger \& Smith, 1986; Bolliger et al., 2000a; Minhas et al., 2000; El-Nagar et al., 2002).

Segundo Varnam \& Sutherland (1994), a viscosidade da calda aumenta como resultado da interação entre o estabilizante e outros solutos, como os açúcares. Além disso, a cristalização da água no processamento e estocagem de sorvetes é afetada pela presença de açúcares, que provocam redução do ponto de congelação (Hartel, 1996). Normalmente, considera-se que apenas $50 \%$ da água do sorvete está congelada à temperatura de fusão, aumentando para $82 \%$ a $-15^{\circ} \mathrm{C}$, e acima de $90 \%$ a $-25^{\circ} \mathrm{C}$ (Hartel, 1998), no entanto esse comportamento depende da formulação. Bolliger et al. (2000b) mostraram que os estabilizantes contribuem para a proteção do sorvete contra a recristalização, em virtude do aumento da viscoelasticidade do produto causado pelo aumento da concentração dos polissacarídeos na fase não congelada do sorvete.

As condições de cristalização ou recristalização da água podem ser afetadas pela Tg' (temperatura de transição vítrea da fração maximamente crioconcentrada) que, por sua vez, também é influenciada pela composição da calda. Segundo Goff et al. (1993), a Tg’ de sorvetes pode variar de $-23^{\circ} \mathrm{C}$ a $-43^{\circ} \mathrm{C}$ dependendo da formulação. Na transição vítrea, o material apresenta alta viscosidade (em torno de $10^{12} \mathrm{~Pa} . \mathrm{s}$ ), sendo caracterizado como um estado metaestável (Levine \& Slade, 1988).

O armazenamento de sorvetes em temperaturas abaixo de Tg' resultaria em não recristalização da água, porém, este procedimento é impraticável. Sorvetes normalmente são armazenados em temperaturas $\left(-10^{\circ} \mathrm{C}\right.$ a $-20^{\circ} \mathrm{C}$ ) acima do ponto de transição vítrea (Hartel, 1998). Além da Tg’, a fração de água não congelada $\left(\mathrm{w}_{\mathrm{nc}}\right)$ pode influenciar a recristalização da água em virtude de flutuações de temperatura durante o armazenamento (Goff, 1992). Esta recristalização é minimizada ao se conseguir manter uma temperatura de estocagem baixa e constante.

O Brasil é um país rico em frutos tropicais exóticos, de alto valor nutricional, presença de vitaminas e pigmentos naturais, e com alto potencial de industrialização, até mesmo na forma de sherbet. A mangaba (Hancornia speciosa) é um desses frutos, cuja industrialização para a produção de sucos, sorvetes e doces, tem sido muito pouco explorada, embora seus produtos elaborados artesanalmente tenham grande aceitação do consumidor na Região Nordeste do Brasil (Seagri, 1994).

O objetivo deste trabalho foi estudar os efeitos das concentrações de goma guar e da polpa de fruta sobre a viscosidade e as transições de fases da calda, e a incorporação de ar no sherbet de mangaba.

\section{Material e Métodos}

Foram estudadas 12 diferentes formulações de mistura para sherbet de mangaba. Cada formulação foi produzida em um único processamento. Cada mistura foi balanceada variando as concentrações de polpa de mangaba (21,1\%, $25,8 \%$ e $28,6 \%)$ e estabilizante goma guar $(0,2 \%, 0,3 \%, 0,4 \%$ e $0,5 \%)$ (Tabela 1$)$, de forma a se obter a mesma concentração em massa de leite em pó (4\%), sacarose (18\%), dextrose (5\%), creme de leite $(2 \%)$ e alginato $(0,3 \%)$, em conformidade com as normas legais vigentes (Anvisa, 1999).

Os ingredientes usados no estudo foram: polpa de mangaba madura (Polpa Fruta Mil Com. de Frutas e Sucos Ltda.); goma guar (Galactomanas, E412, Colóides Naturels Brasil Comercial Ltda.); leite em pó e creme de leite (Nestlé Brasil Ltda.); sacarose (Dedini Açúcar e Álcool Ltda.); dextrose desidratada (Corn Products do Brasil); e emulsificante alginato protanal éster BV 4830 (FMC Biopolymer - FMC Corporation, PA, USA).

A calda foi homogeneizada em liquidificador industrial $\left(5^{\circ} \mathrm{C}\right)$ por $5 \mathrm{~min}$, pasteurizada a $70^{\circ} \mathrm{C}$ por $30 \mathrm{~min}$ (Anvisa, 1999), resfriada a $4 \pm 1^{\circ} \mathrm{C}$ e mantida nesta temperatura por 16 horas para maturação. A seguir, adicionou-se a polpa de mangaba pasteurizada. Amostras da calda foram retiradas para determinação da viscosidade e das propriedades térmicas por calorimetria de varredura diferencial (DSC). 
Os sherbets foram preparados em batelada em um trocador de calor horizontal de superfície raspada (Brasfrio PHB 80/100). A temperatura de batimento $\left(-6^{\circ} \mathrm{C}\right)$ foi fixa durante o processamento, assim como a velocidade de rotação das pás (345 rpm). Amostras foram tomadas para medida da incorporação de ar antes e depois do batimento. Os sherbets foram embalados em caixas com capacidade para $10 \mathrm{~L}$, de papel revestido de plástico, a fim de evitar o encolhimento do produto, e armazenados em freezer horizontal com termostato regulado a $-30^{\circ} \mathrm{C}$.

A viscosidade aparente das diferentes misturas para sherbets foi medida, utilizando-se um viscosímetro Brookfield LDVII com cilindros concêntricos. As medidas, feitas em triplicatas, foram realizadas em temperatura constante de $6 \pm 1^{\circ} \mathrm{C}$, com "spindles" 3 e 4 , em velocidade fixa de $30 \mathrm{rpm}$.

As amostras da calda de sherbet foram analisadas com um calorímetro diferencial de varredura DSC-TA2010 controlado por um módulo TA5000. As amostras (em torno de $10 \mathrm{mg}$ ), pesadas em balança de precisão $( \pm 0,01 \mathrm{mg})$, foram acondicionadas em cadinhos herméticos e aquecidas a $10^{\circ} \mathrm{C}$ por minuto, entre $-120^{\circ} \mathrm{C}$ e $100^{\circ} \mathrm{C}$, em atmosfera inerte $\left(45 \mathrm{~mL}\right.$ de $\mathrm{N}_{2}$ por min). A referência foi um cadinho hermético TA de alumínio. Antes das análises, a célula do DSC foi congelada com nitrogênio líquido.

A temperatura de transição vítrea da fração maximamente crioconcentrada ( $\left(g^{\prime}\right.$ ') foi determinada como o ponto de inflexão da linha de base dos termogramas, e a entalpia de fusão $\left(\Delta \mathrm{H}_{\mathrm{f}}\right)$ foi calculada como a área sobre o pico endotérmico (Sobral et al., 2001). Essas determinações foram realizadas com auxílio do software Universal Analysis V1.7F (TA Instruments).

A quantidade de água não congelada $\left(\mathrm{w}_{\mathrm{nc}}\right)$ nas amostras foi calculada pela seguinte equação: $\mathrm{w}_{\mathrm{nc}}=\mathrm{x}_{\mathrm{bu}}-\Delta \mathrm{H}_{\mathrm{f}} / \lambda$, em que $\mathrm{x}_{\mathrm{bu}}$ é a umidade da amostra; $\Delta \mathrm{H}_{\mathrm{f}}$ é a entalpia de fusão; $\lambda$ é o calor latente $\left(333,55 \mathrm{~J} \mathrm{~g}^{-1}\right)$ de fusão da água pura. A umidade das amostras $\left(\mathrm{x}_{\mathrm{bu}}\right)$ foi determinada por secagem em estufa a vácuo, a $70^{\circ} \mathrm{C}$, até peso constante (Cecchi, 1999).

A incorporação de ar (IAr) nos sherbets foi calculada pela relação entre a massa do sherbet e a massa da calda antes do batimento, com a seguinte equação:

$\mathrm{IAr}=($ massa de sherbets - massa da mistura $) /$ massa da mistura.

Na comparação entre as médias das propriedades de fases de cada concentração de polpa, com concentração de estabilizantes variável (Tabela 1), e das médias das medidas realizadas em triplicatas para a incorporação de ar (Tabela 2), considerando-se os 12 ensaios, empregou-se o teste de média de Tukey.

\section{Resultados e Discussão}

A viscosidade das caldas de sherbets variou de 85,8 a 286,1 cP, aumentando progressivamente com o aumento da concentração de estabilizante (Figura 1). Este

Tabela 1. Umidade $\left(\mathrm{x}_{\mathrm{bu}}\right)$, entalpia de fusão $\left(\Delta \mathrm{H}_{\mathrm{f}}\right)$, quantidade de água não congelada $\left(\mathrm{w}_{\mathrm{nc}}\right)$, temperatura de fusão $\left(\mathrm{T}_{\mathrm{f}}\right)$ e temperatura de transição vítrea da fração maximamente crioconcentrada (Tg’) das misturas para sherbets com diferentes concentrações de goma guar e polpa de mangaba(1).

\begin{tabular}{|c|c|c|c|c|c|c|c|}
\hline Mangaba $\left(\mathrm{g} 100 \mathrm{~g}^{-1}\right)$ & Goma guar $\left({\left.\mathrm{g} 100 \mathrm{~g}^{-1}\right)}\right.$ & $\mathrm{x}_{\mathrm{bu}}\left(\mathrm{g} \mathrm{g}^{-1}\right)$ & $\Delta \mathrm{H}_{\mathrm{f}}\left(\mathrm{J} \mathrm{g}^{-1}\right)$ & $\mathrm{W}_{\mathrm{nc}}\left(\mathrm{g} \mathrm{g} \mathrm{g}^{-1}\right)$ & $\mathrm{w}_{\mathrm{nc}} / \mathrm{x}_{\mathrm{bu}}(\%)$ & $\mathrm{T}_{\mathrm{f}}\left({ }^{\circ} \mathrm{C}\right)$ & Tg' $\left({ }^{\circ} \mathrm{C}\right)$ \\
\hline 21,1 & 0,2 & 0,701 & 188,0 & 0,137 & 20 & $-0,77$ & $-57,2$ \\
\hline 21,1 & 0,3 & 0,665 & 184,5 & 0,112 & 17 & $-1,13$ & $-53,5$ \\
\hline 21,1 & 0,4 & 0,690 & 196,2 & 0,102 & 15 & $-0,84$ & $-55,7$ \\
\hline 21,1 & 0,5 & 0,687 & 195,5 & 0,101 & 15 & $-1,04$ & $-56,0$ \\
\hline Média & & $\begin{array}{r}0,686 a \\
( \pm 0,015)\end{array}$ & $\begin{array}{l}191,1 \mathrm{~b} \\
( \pm 5,73)\end{array}$ & $\begin{array}{r}0,113 a \\
( \pm 0,017)\end{array}$ & $\begin{array}{c}17 a \\
( \pm 2,36)\end{array}$ & $\begin{array}{c}-0,95 \\
( \pm 0,17)\end{array}$ & $\begin{array}{l}-55,6 a \\
( \pm 1,6)\end{array}$ \\
\hline 25,8 & 0,2 & 0,695 & 199,0 & 0,098 & 14 & $-0,45$ & $-56,5$ \\
\hline 25,8 & 0,3 & 0,685 & 192,7 & 0,107 & 16 & $-1,16$ & $-56,7$ \\
\hline 25,8 & 0,4 & 0,670 & 194,8 & 0,086 & 13 & $-1,00$ & $-56,0$ \\
\hline 25,8 & 0,5 & 0,680 & 196,4 & 0,091 & 13 & $-0,29$ & $-56,1$ \\
\hline Média & & $\begin{array}{r}0,683 a \\
( \pm 0,010)\end{array}$ & $\begin{array}{r}195,7 b \\
( \pm, 66)\end{array}$ & $\begin{array}{c}0,096 \mathrm{ab} \\
( \pm 0,009)\end{array}$ & $\begin{array}{c}14 \mathrm{ab} \\
( \pm 1,41)\end{array}$ & $\begin{array}{c}-0,73 \\
( \pm 0,42)\end{array}$ & $\begin{array}{l}-56,3 a \\
( \pm 0,4)\end{array}$ \\
\hline 28,6 & 0,2 & 0,711 & 208,7 & 0,085 & 12 & $-0,61$ & $-56,2$ \\
\hline 28,6 & 0,3 & 0,703 & 206,5 & 0,084 & 12 & $-0,68$ & $-56,7$ \\
\hline 28,6 & 0,4 & 0,689 & 202,2 & 0,083 & 12 & $-1,19$ & $-56,8$ \\
\hline 28,6 & 0,5 & 0,709 & 207,2 & 0,088 & 12 & $-0,72$ & $-57,7$ \\
\hline Média & & $\begin{array}{r}0,703 a \\
( \pm 0,009)\end{array}$ & $\begin{array}{l}206,2 \mathrm{a} \\
( \pm 2,79)\end{array}$ & $\begin{array}{r}0,085 b \\
( \pm 0,002)\end{array}$ & $\begin{array}{c}12 \mathrm{~b} \\
( \pm 0,00)\end{array}$ & $\begin{array}{c}-0,80 \\
( \pm 0,26)\end{array}$ & $\begin{array}{l}-56,8 \mathrm{a} \\
( \pm 0,6)\end{array}$ \\
\hline
\end{tabular}

(1)Em uma mesma coluna, médias com letras desiguais diferiram significativamente entre si pelo teste de Tukey a 5\% de probabilidade. 
fenômeno foi também observado por Wittinger \& Smith (1986), Goff et al. (1993), Bolliger et al. (2000a), Minhas et al. (2000) e Kaya \& Tekin (2001). Pôde-se, ainda, observar que a concentração de polpa de mangaba influenciou o aumento da viscosidade. Esse aumento foi mais importante nas caldas contendo $28,6 \%$ de polpa, principalmente no domínio de altas concentrações de estabilizante $(0,4 \%$ e $0,5 \%)$. Diferenças $(\mathrm{P}>0,05)$ não foram observadas entre as amostras com $0,2 \%$ de estabilizante. Esse comportamento está de acordo com os resultados obtidos por Guven \& Karaca (2002), que observaram um aumento da viscosidade aparente de misturas para sorvete de iogurte quando maior quantidade de polpa de morango era adicionada, mantendo-se uma concentração fixa de estabilizante. Maiores concentrações de polpa empregadas nas formulações proporcionam aumento da concentração de sólidos solúveis. Desta forma, a quantidade de água livre na mistura diminui, aumentando, assim, a viscosidade e potencializando o efeito estabilizante (Marshall \& Arbuckle, 1996). Além disso, a presença de polissacarídeos na polpa, como pectina e celulose, contribui para o aumento da viscosidade.

Exemplos de termogramas obtidos nas análises térmicas da calda para sherbets estão apresentados na Figura 2a, em que se pode observar dois fenômenos: uma transição vítrea e a fusão do gelo, na forma de um endoterma. A observação do desvio da linha de base na transição vítrea é prejudicada pela endoterma, que envolve variação de energia muito superior à transição vítrea (Sobral et al., 2001). Entretanto, esse fenômeno pode ser melhor visualizado em escala ampliada, conforme o encarte apresentado na Figura 2b.

Tabela 2. Valores médios de incorporação de ar para os sherbets preparados com diferentes concentrações de polpa de mangaba e goma guar ${ }^{(1)}$.

\begin{tabular}{|c|c|c|c|}
\hline $\begin{array}{l}\text { Mangaba } \\
\left({\left.\mathrm{g} 100 \mathrm{~g}^{-1}\right)}\right.\end{array}$ & $\begin{array}{l}\text { Goma guar } \\
\left({\left.\mathrm{g} 100 \mathrm{~g}^{-1}\right)}\right.\end{array}$ & $\begin{array}{c}\text { Incorporação de ar } \\
(\%)\end{array}$ & $\sigma^{(2)}$ \\
\hline 21,1 & 0,2 & $34,33 \mathrm{c}$ & 0,18 \\
\hline 21,1 & 0,3 & $26,39 \mathrm{e}$ & 0,01 \\
\hline 21,1 & 0,4 & $22,58 \mathrm{f}$ & 0,03 \\
\hline 21,1 & 0,5 & $36,96 \mathrm{~b}$ & 0,05 \\
\hline 25,8 & 0,2 & $20,01 \mathrm{~g}$ & 0,50 \\
\hline 25,8 & 0,3 & $26,56 \mathrm{e}$ & 0,12 \\
\hline 25,8 & 0,4 & $37,17 \mathrm{~b}$ & 0,02 \\
\hline 25,8 & 0,5 & $40,59 \mathrm{a}$ & 0,04 \\
\hline 28,6 & 0,2 & $26,39 \mathrm{e}$ & 0,12 \\
\hline 28,6 & 0,3 & $26,63 \mathrm{e}$ & 0,06 \\
\hline 28,6 & 0,4 & $27,53 \mathrm{~d}$ & 0,02 \\
\hline 28,6 & 0,5 & $22,22 \mathrm{f}$ & 0,03 \\
\hline
\end{tabular}

(1)Em uma mesma coluna, médias com letras desiguais diferiram significativamente entre si pelo teste de Tukey a $5 \%$ de probabilidade. (2)Desvio-padrão das médias $(\mathrm{n}=3)$.
Esses resultados são típicos de sistemas com muita água, e podem ser observados em caldas de sorvetes (Goff et al., 1993), soluções diluídas de sacarose (Roos \& Karel, 1991) e polpas de frutas (Sobral et al., 2001; Telis \& Sobral, 2002). De acordo com Goff (1994), essa transição vítrea em sistemas muito úmidos corresponde a Tg', a transição vítrea da fração maximamente concentrada que, além disso, é insensível à variação da umidade da amostra, acima de 50\% de umidade, mas sensível à composição da matéria seca.

As propriedades físico-químicas calculadas a partir dos termogramas são apresentadas na Tabela 1. Observou-se que a calda produzida com a maior concentração de polpa de mangaba (28,6\%) apresentou maior $(\mathrm{P} \leq 0,05)$ valor médio da entalpia de fusão do gelo $\left(\Delta \mathrm{H}_{\mathrm{f}}\right)$. De maneira geral, a entalpia de fusão do gelo aumenta com a umidade do produto (Telis \& Sobral, 2002). Dessa maneira, o comportamento de $\Delta \mathrm{H}_{\mathrm{f}}$, neste trabalho, deve ter sido conseqüência da variação da umidade das amostras, embora não tenha sido observada diferença significativa $(\mathrm{P} \geq 0,05)$ entre os valores da umidade das amostras. Valores médios da $\Delta \mathrm{H}_{\mathrm{f}}$ das amostras com mesma concentração de estabilizantes não foram significativamente diferentes $(\mathrm{P} \geq 0,05)$.

Observa-se, na Tabela 1, uma diminuição significativa $(\mathrm{P} \leq 0,05)$ da fração de água não congelada $\left(\mathrm{w}_{\mathrm{nc}}\right)$ entre as amostras com $21,1 \%$ e $28,6 \%$ de polpa de fruta. Esse resultado se deve ao aumento da umidade, ocasionado pela adição de maior quantidade de polpa e, con-

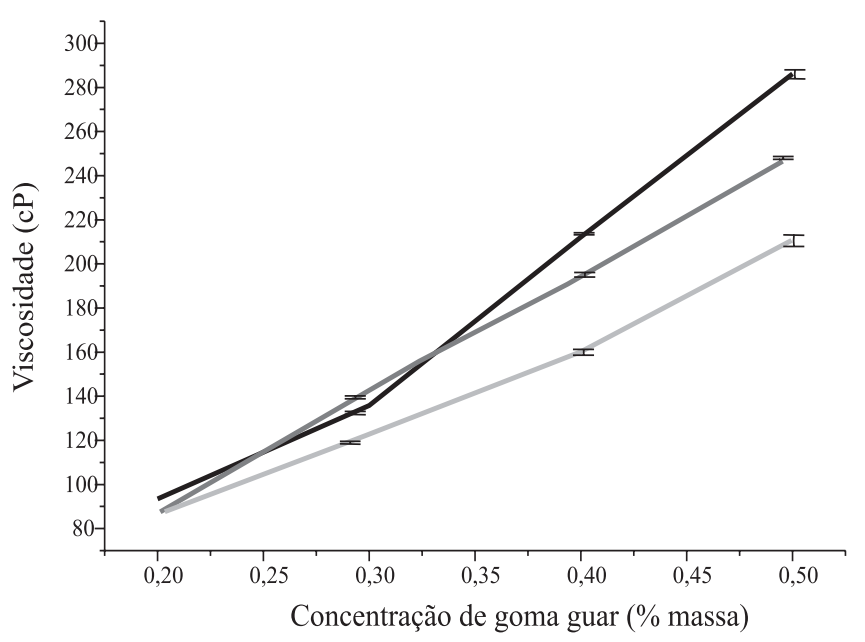

Figura 1. Viscosidade aparente das amostras de caldas de sherbets em razão da concentração de estabilizante para diferentes concentrações de polpa de mangaba com média de $21,1 \%(-), 25,8 \%(-)$ e $28,6 \%(-)$. 
seqüentemente, à diminuição do teor de sólidos solúveis. A diluição da amostra justifica o aumento do calor latente de fusão, da fração de água não congelável e da temperatura de fusão.

A temperatura de fusão do gelo $\left(\mathrm{T}_{\mathrm{f}}\right)$ mostrou tendência a aumentar com maior adição da polpa de fruta, muito possivelmente por causa do aumento da umidade das amostras, cujo efeito deve ter sido superior ao do acréscimo do açúcar da polpa. Essa propriedade é pouco influenciada por biopolímeros, como a goma guar. A temperatura de transição vítrea da fração maximamente crioconcentrada (Tg') não foi afetada pela adição da polpa (Tabela 1). Hartel (1998) determinou valores da $\mathrm{Tg}$ ' para sorvete de baunilha, entre $-32^{\circ} \mathrm{C}$ e $-35^{\circ} \mathrm{C}$, muito superiores aos deste trabalho, cujo valor médio foi $-56,24^{\circ} \mathrm{C}$.

Normalmente, a Tg' é influenciada por biopolímeros, que tendem a aumentar o valor dessa propriedade, e por isso são usados como crioprotetores (Slade \& Levine, 1991). Entretanto, a faixa de concentração de goma guar adicionada às misturas para sherbets, estudada neste experimento (de 0,2 a 0,5 g $100 \mathrm{~g}^{-1}$ de calda) (Tabela 1) não foi suficiente para se perceber esta influência.

Os resultados das análises de IAr são apresentados na Tabela 2, em que se observa valores dentro da faixa esperada para sherbets, isto é, entre $25 \%$ e $50 \%$ de ar incorporado na massa (Marshall \& Arbuckle, 1996). Nas formulações com 25,8\% de polpa, foi observada uma influência positiva e linear $\left(r^{2}=0,98\right)$ da concentração do estabilizante sobre a IAr dos sherbets. O aumento da incorporação de ar com a adição de estabilizante era esperado pela maior quantidade de micelas formadas, entretanto este efeito não foi observado em outras formulações, com $21,1 \%$ e $28,6 \%$ de polpa.

A amostra que apresentou maior $\operatorname{IAr}(40,59 \%)$ foi a que continha $25,8 \%$ de polpa e $0,4 \%$ de goma guar, sendo que a de menor valor continha a mesma concentração de polpa, porém com $0,1 \%$ de estabilizante. Os valores obtidos nos ensaios 2, 6 e 10 não diferiram significativamente $(\mathrm{P}>0,05)$, mostrando que as amostras com $0,2 \%$ de goma guar não apresentaram variações neste parâmetro quando três diferentes concentrações de polpa de mangaba foram usadas (Tabela 2).
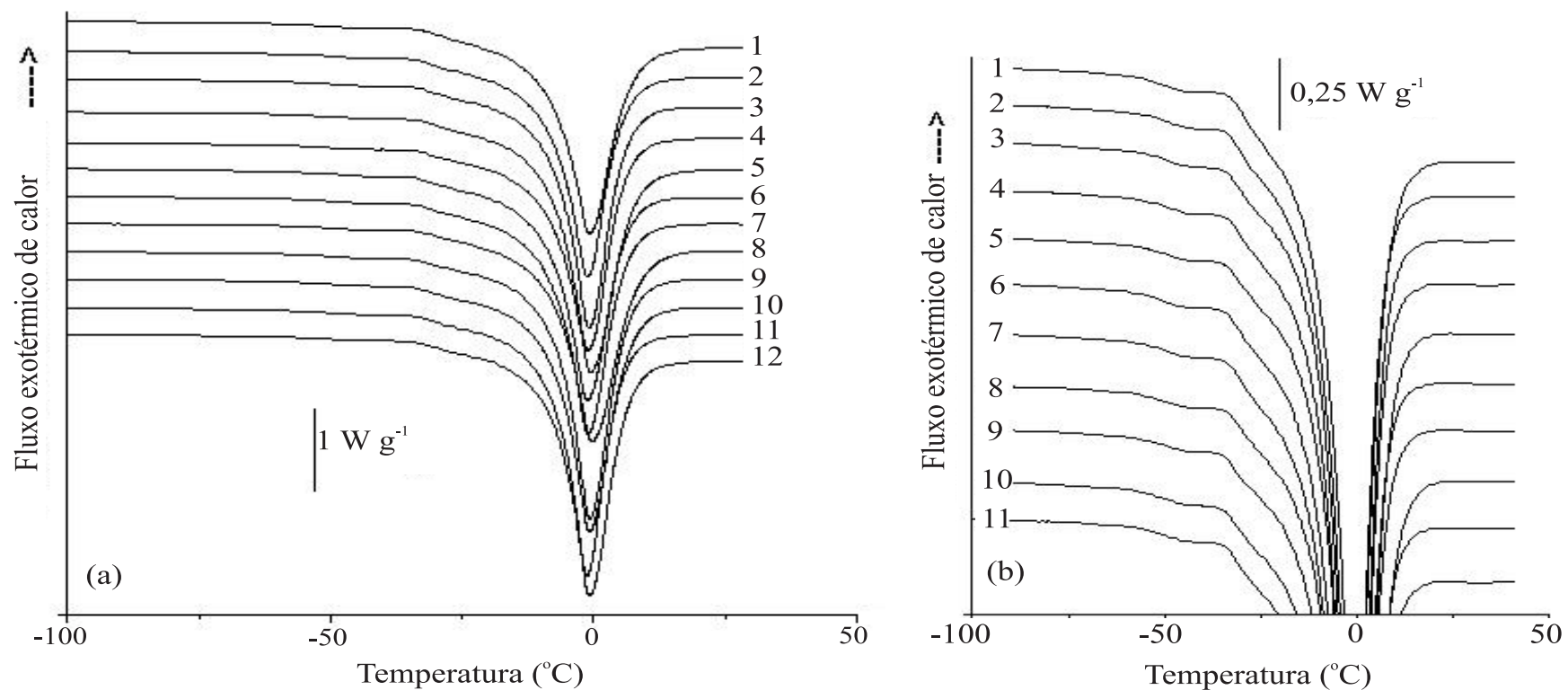

Figura 2. Termogramas representativos (a) e termogramas ampliados (b) na região da transição vítrea das 12 amostras de caldas de sherbets: 1, 2, 3 e 4 (21,1\% polpa); 5, 6, 7 e 8 (25,8\%); 9, 10, 11 e 12 (28,6\%). 


\section{Conclusões}

1. Maiores concentrações de goma guar e polpa de mangaba aumentam a viscosidade da mistura para sherbets.

2. O aumento na concentração da polpa de fruta provoca diminuição da fração de água não congelada.

3. O aumento da incorporação de ar no sherbets, associado ao aumento da concentração de estabilizante, é observado apenas nas misturas com 25,8\% de polpa de mangaba.

\section{Agradecimentos}

À Colóides Naturels Brasil Comercial Ltda., Nestlé Brasil Ltda., Dedini Açúcar e Álcool Ltda., SP, Brasil, Corn Products do Brasil, SP, Brasil e FMC Corporation, PA, USA, pelo fornecimento de matéria-prima; à Prefeitura do Campus Administrativo da USP de Pirassununga, pelo apoio.

\section{Referências}

ANVISA, Agência Nacional de Vigilância Sanitária. Portaria nº 379, de 26 de abril de 1999. Disponível em: <http://www.anvisa.gov.br/ legis/portarias/379_99.htm>. Acesso em: jan. 2003.

BOLLIGER, S.; KORNBRUST, B.; GOFF, H.D.; THARP, H.D.; WINDHAB, E.J. Correlation between colloidal properties of ice cream mix and ice cream. International Dairy Journal, v.10, p.303309, 2000b.

BOLLIGER, S.; KORNBRUST, B.; GOFF, H.D.; THARP, H.D.; WINDHAB, E.J. Influence of emulsifiers on ice cream produced by conventional freezing and low-temperature extrusion processing. International Dairy Journal, v.10, p.497-504, 2000a.

CECCHI, M. Fundamentos Teóricos e Práticos em Análise de Alimentos. Campinas: Editora da Unicamp, 1999, p.212.

CHANG, Y.; HARTEL, R.W. Development of air cells in a batch ice cream freezer. Journal of Food Engineering, v.55, p.71-78, 2002.

EL-NAGAR, G.; CLOWES, G.; TUDORICÃ, C.M.; KURI, V. Rheological quality and stability of yog-ice cream with added inulin. International Journal of Dairy Technology, v.55, p.89-93, 2002.

GOFF, H.D. Low-temperature stability and the glassy state in frozen foods. Food Research International, v.25, p.317-325, 1992.

GOFF, H.D Measuring and interpreting the glass transition in frozen foods and model systems. Food Research International, v.27, p.187-189, 1994.

GOFF, H.D.; CALDWELL, K.B.; STANLEY, D.W. The influence of polysaccharides on the glass transition in frozen sucrose solutions and ice cream. Journal of Dairy Science, v.76, p.1268-1277, 1993.
GUVEN, M.; KARACA, O.B. The effects of varying sugar content and fruit concentration on the physical properties of vanilla and fruit ice-cream-type frozen Yogurts. International Journal of Dairy Technology, v.55, p.27-31, 2002.

HARTEL, R.W. Ice crystalization during the manufacture of ice cream. Trends in Food Science \& Technology, v.7, p.315-321, 1996.

HARTEL, R.W. Phase Transition in Ice Cream. In: RAO, M.A.; HARTEL, R.W. (Ed.). Phase/state transition in foods: chemical, structural and rheological changes. New York: Marcel Dekker, 1998. p.327-368.

INNOCENTE, N.; CAMPARIN, D.; CORRADINI, C. Proteosepeptone whey fraction as emulsifier in ice-cream preparation. International Dairy Journal, v.12, p.69-74, 2002.

KAYA, S.; TEKIN, A.R. The effect of salep content on the rheological characteristics of a typical ice-cream mix. Journal of Food Engineering, v.47, p.59-62, 2001.

LEVINE, H.; SLADE, L. Principles of cryo-stabilization technology from structure/property relationships of carbohydrate/water systems: a review. CryoLetters, v.9, p.21-63, 1988.

MARSHALL, R.T.; ARBUCKLE, W.S. Ice cream. $5^{\text {th }}$ ed. New York: Chapman \& Hall, 1996. 349p.

MINHAS, K.S.; SIDHU, J.S.; MUDAHAR, G.S.; SINGH, A.K. Effect of different concentrations of stabilizers and ageing times on the viscosity of plain ice cream mix made from buffalo milk. Journal of Food Science and Technology, v.37, p.602-608, 2000.

REID, D.S. Crystalization Phenomena in the Frozen State. In: RAO, M.A.; HARTEL, R.W. (Ed.). Phase/state transition in Foods: chemical, structural and rheological changes. New York: Marcel Dekker, 1998. p.313-325.

ROOS, Y.; KAREL, M. Phase transitions of amorphous sucrose and frozen sucrose solutions. Journal of Food Science, v.56, p.266267, 1991.

SEAGRI - Secretaria de Agricultura, Irrigação e Reforma Agrária. Cultura-Mangaba. Disponível em: <http://www.seagri.ba.gov.br/ Mangaba.htm>. Acesso em: mar. de 2003.

SLADE, L.; LEVINE, H. Beyond water activity: recent advances based on an alternative approach to the assessment of food quality and safety. Critical Reviews in Food Science and Nutrition, v.30, p.115-360, 1991.

SOBRAL, P.J.A.; TELIS, V.R.N.; HABITANTE, A.M.Q.B.; SERENO, A. Phase diagram for freeze-dried persimmon. Thermochimica Acta, v.376, p.83-89, 2001.

TELIS, V.R.N.; SOBRAL, P.J.A. Glass transitions for freeze-dried and air-dried tomato. Food Research International, v.35, p.435443, 2002.

VARNAM, A.H.; SUTHERLAND, J.P. Milk and Milk Products, v.1. London: Chapman \& Hall, 1994. p.386-437.

WITTINGER, S.A.; SMITH, D.E. Effect of sweetener/stabilizer interaction on the viscosity and freezing point of ice cream mix. Milchwissenchaft, v.41, p.766-769, 1986.

Recebido em 14 de abril de 2004 e aprovado em 1ํ de fevereiro de 2005 